\title{
Planetary exploration
}

\section{Europe and the large solar system projects}

\author{
Hans Balsiger
}

Europe made great advances in planetary exploration with the space probe to Halley's comet. The current and future plans incorporated in the Horizon 2000 core programme are minimal but balanced. They are currently threatened by lack of resources: it would be unforgivable to leave this field to the large space powers now.

Hans Balsiger is Professor of Physics, Physikalisches Institut, Universităt Bern, Sidlerstrasse 5, CH-3012 Bern, Switzerland.
OINCE SOLAR SYSTEM science began in the early 17 th century, when some of the more spectacular discoveries were made by the telescope and when, for example Descartes proposed a remarkably accurate view on the formation of the Sun and the planets; European scientists have played an important role in this field. In the 50 s a strong community developed which analysed with novel instrumentation the only samples available from space, the meteorites. This same community made important contributions to the analysis of lunar samples provided by the Apollo astronauts and by the unmanned USSR lunar missions. Its expertise will again be called upon when samples from Mars and from a comet will hopefully become available shortly after the turn of the century.

The space age of solar system exploration began in 1962 with the Venus flyby of Mariner-2, and here Europeans had to assume the role of junior partners in the programmes of the US and USSR space agencies. The lack of its own launcher and the relatively small budget restricted the European Space Agency (ESA) to space missions close to the Earth with payloads dedicated to astronomy or magnetosphere/solar wind science.

Several attempts were made to get a planetary mission approved within ESA, but it took until 1980 for the now well-known Giotto mission to Comet Halley to be selected. In the meantime, several national programmes had profited from the generous hospitality of the two space powers and thus were able to fly experiments on planetary missions and to gain experience in this field.

In this presentation some highlights of the first and highly successful European deep space mission to Comet Halley are discussed, and a pre- 
view is given of future ESA solar system missions which are already scheduled or in the planning phase.

For the present purpose solar system exploration is divided into three fields:

- the study of planetary bodies, including moons, asteroids, comets, meteorites, and dust;

- the investigation of the plasmas in the solar system, that is the solar wind, the planetary magnetospheres, including their interaction with the solar wind;

- the study of the Sun itself, including the source region of the solar wind, the corona.

In the first field, the main European expertise until recently has been in the laboratories where meteorites and lunar samples have been, and are still, analysed. These laboratories are very competitive world-wide, and they would have the expertise to make important contributions when Mars and cometary material is returned to earth. In addition, of course, Europe has recently gained a head start in cometary chemistry and physics with its Giotto mission to Halley's comet.

In the second field, European scientists have also managed to make important contributions. Firstly, based on the facts that such investigations do not necessarily need deep space probes (Earth's magnetosphere) and that they are somewhat less complex than those for planetary explorations, it took Europe's scientists a relatively short time to catch up with their colleagues. In some cases they even managed to take the lead in developing new techniques (for instance in the measurements of electric fields by tracking electron beams or in the analysis of plasma composition).

Secondly, the Scandinavian countries have a long history of research in auroral phenomena and have, since the beginning of the space age, been in the forefront of all investigations relating to aurorae. Hence, research of the terrestrial upper atmosphere and magnetosphere, both experimental and theoretical, has been strongly developed.

For the third field there has also always been a broad interest among ground based observers in Europe. With its first cornerstone ESA will in the near future make its space-based contribution in

\section{Giotto was able to cross the contact surface of Halley's comet and it allowed the gas and ion mass spectrometers to analyse the inner coma, the atmosphere/ionosphere, which was undisturbed by the solar wind}

the form of the Solar and Heliospheric Observatory, SOHO. It is especially noteworthy that this observatory will include the most complete payload for helioseismology to date. This technique allows the investigation of the inner structure of the Sun and will thus greatly advance our understanding of the physics of this our nearest star.

I shall concentrate on the planetary exploration. It is the most costly, because of its need of deep space probes, and it calls for large missions.

\section{Past planetary exploration}

For a long time it looked as if an independent planetary exploration would be out of reach for European laboratories and scientists. They learned their trade as guest investigators on spacecraft of the big space powers, USA and USSR. Scientists could contribute experiments or were co-investigators on several planetary missions including the very spectacular Voyager missions. Only in 1980 did ESA select its first, and up to now only, independent planetary mission, the one to comet $\mathrm{Hal}$ ley. This fortunate event came about because NASA cancelled the originally planned joint NASA/ESA mission to comets Halley and Temple-2.

Between 1982 and 1984 a Joint Working Group (JWG) of the Space Science Committee of the European Science Foundation and the Space Science Board of the US National Academy of Sciences studied a joint programme of planetary explorations based on equal partnership. Many of the recommendations of the JWG have been taken up by the agencies, in particular the Titan Probe/Saturn Orbiter mission (now called Cassini/ Huygens). But for Europe and ESA the most important development in the field occurred when the plan for a long-term strategy was born.

The so-called Horizon 2000 was built around four cornerstones, two for astronomy and two for solar systems science, with smaller missions in between as the flexible element. While one of the solar system cornerstones was dedicated to solar terrestrial physics and thus includes the previously mentioned fields two and three, the other would be a true planetology mission aiming at the return of cometary material to earth.

Horizon 2000 was acknowledged by the ministerial conference in Rome 1985 as the minimum science programme of ESA. Whereas the financial framework for the core programme was basically established, it was also recognised that some of the more elaborate missions of the future, such as the European contribution to a Mars mission, would not fit into this framework and would have to find supplementary financial support.

Let me now come to a short presentation of ESA's programme in planetary science. As mentioned before, the only European planetary 


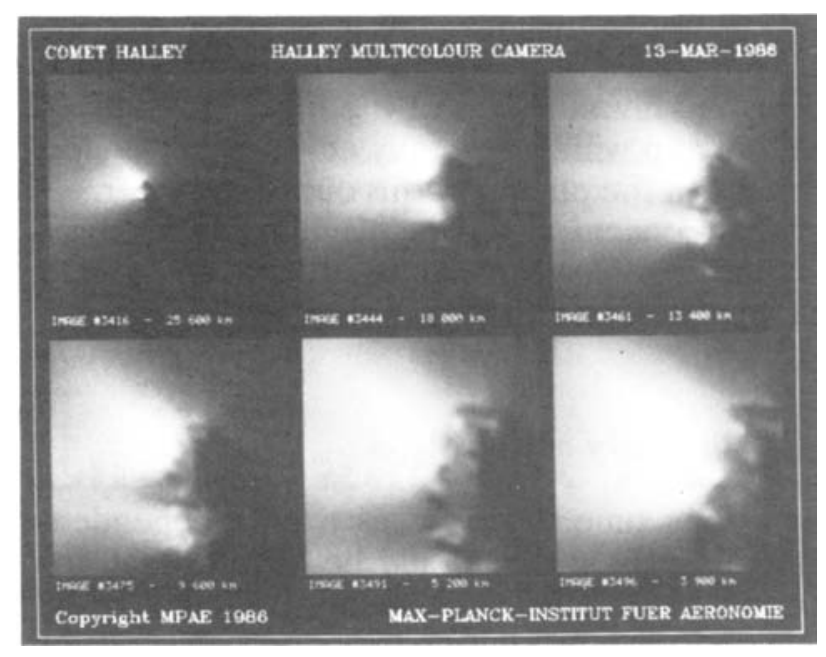

Figure 1. Views of Comet Halley taken during the fly-by of the European Glotto probe

Note: The source regions of the dust and gas jets on the sunlit side of the comet cover only about $10 \%$ of the comet surface

mission in the past was Giotto, the fly-by at Halley's comet on 13/14 March 1986. Of all the international fleet of spacecraft, Giotto was the only probe equipped with a dust shield and it could therefore dare to approach the comet much closer than the others $(600 \mathrm{~km}$, compared to $8,000 \mathrm{~km}$ of the second closest, the Russian VEGA-2 mission).
Thus Giotto was able to cross the so-called contact surface or ionopause of the comet and it allowed the gas and ion mass spectometers to analyse the inner coma, the comet's atmosphere/ionosphere, which is undisturbed by the solar wind. These analyses demonstrated that comets are indeed the most primitive bodies in the solar system analysed so far, which have retained a lot of the volatile material $(80 \%$ water, surprisingly low amounts of ammonia, methane, and cyanide). Most of these volatiles have been lost in the inner planets, the moon, and in the meteorites.

Halley's chemical and isotopic composition was found to be very similar to solar material with possibly some resemblance to the solar system's ancestor, an unnamed dark interstellar cloud. The spectacular pictures taken by the camera (Figure 1) also revealed the darkest object known so far in the solar system, a potato shaped nucleus with gas and dust jets coming out of only about $10 \%$ of its surface.

\section{Present and future}

Giotto also brings us to the present and near future. The spacecraft with about $50 \%$ of the payload functioning well was tested at the beginning of 1990 , and has subsequently been ejected towards comet Grigg-Skjellerup by means of a close Earth

\section{ALTITUDE

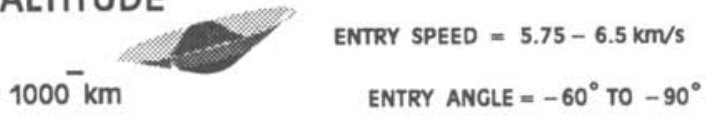

\section{TITAN HUYGENS PROBE ENTRY AND DESCENT SCENARIO}

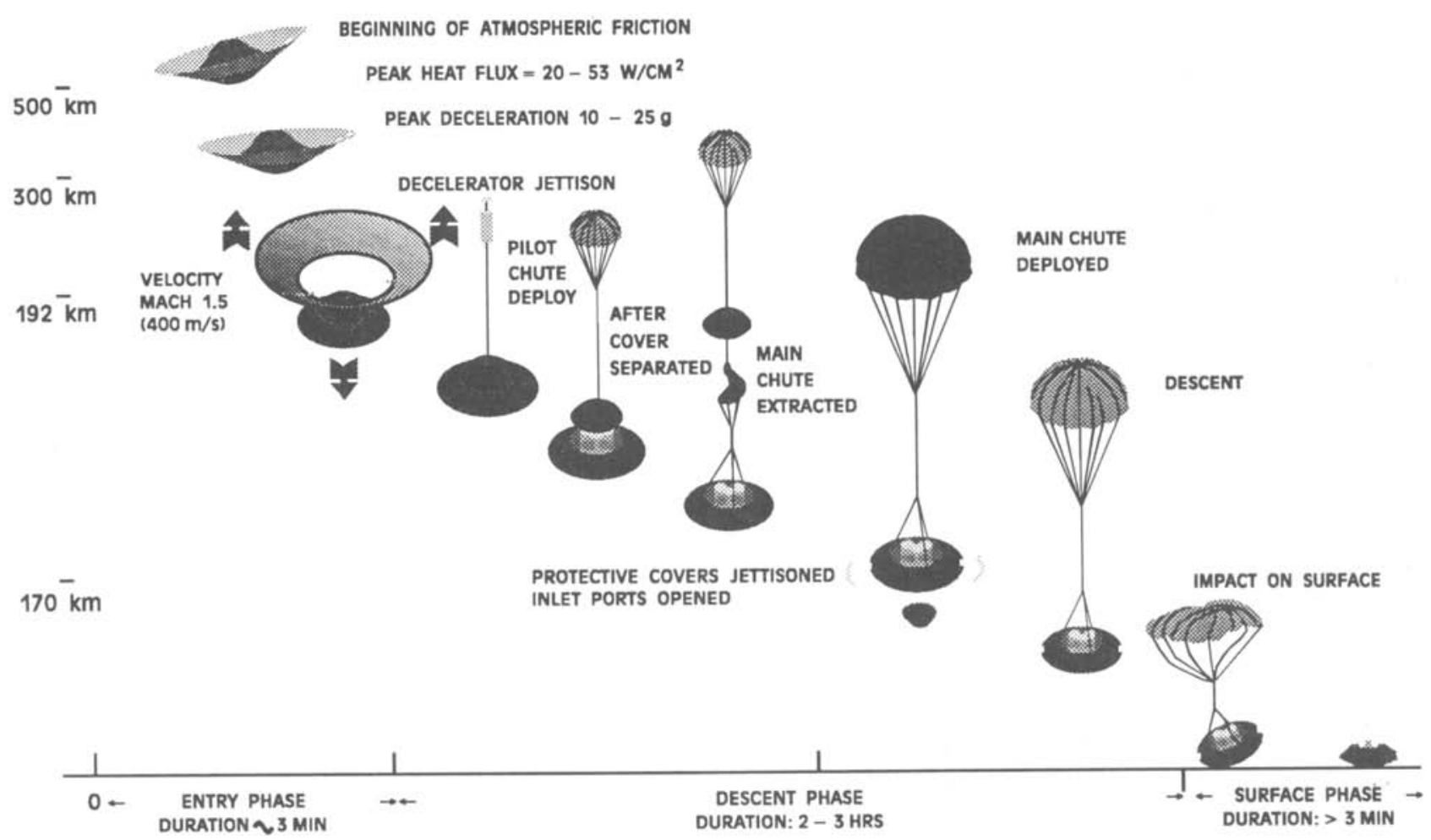

Figure 2. Scenario for the European Huygens probe descending to the surface of Saturn's moon Titan 
fly-by. This feat by the European Operations Centre in Darmstadt was the first such gravityassisted manoeuvre ever performed with a deepspace-probe at the Earth. The new Giotto Extended Mission thus is on its way to investigate the solar wind interaction of an older, much less active comet than Halley.

The next planetary mission will be Cassini/Huygens, a collaborative mission with NASA which includes a Saturn orbiter and a Titan probe. The European part, selected as a medium sized mission from the flexible part of Horizon 2000 , will be the small but very interesting Huygens probe which will descend through Titan's atmosphere to its surface. The launch is scheduled for 1996 but the spacecraft will arrive at Saturn only 6.5 years later, in 2002.

The probe with its combined aerobrake and parachute system (see Figure 2) should be a great challenge for the European industry. The experimental package which has to survive and to perform analyses in the quite hostile environment of Titan's primitive and cold atmosphere (90-170 $\mathrm{K})$ should be a similar challenge to the scientists.
The descent phase will last 2-3 hours, and it is hoped that the package will stay alive on Titan's icy or liquid ethane surface $(90 \mathrm{~K})$ for more than two minutes.

For the beginning of the next century ESA plans to launch its planetary cornerstone mission Rosetta. It is presently being studied as a joint mission with NASA, and it aims at the return of cometary samples to Earth and subsequent analysis in laboratories. As for the Moon, it is recognised that a thorough structural, chemical, and isotopic analysis of cometary material can only be performed in the laboratory and not remotely. Of course, the mission includes supporting measurements during the approach phase and at the landing site; however, its main goal is the return to Earth of a three meter drill core of cometary material in as undisturbed a condition as possible. The scenario to achieve that is, of course, quite complex and challenging. Its main phases are described in Figure 3.

Even further in the future are unmanned and manned missions to Mars and/or the establishment of a lunar base. ESA is not able by itself to compete independently for these endeavours; however, it

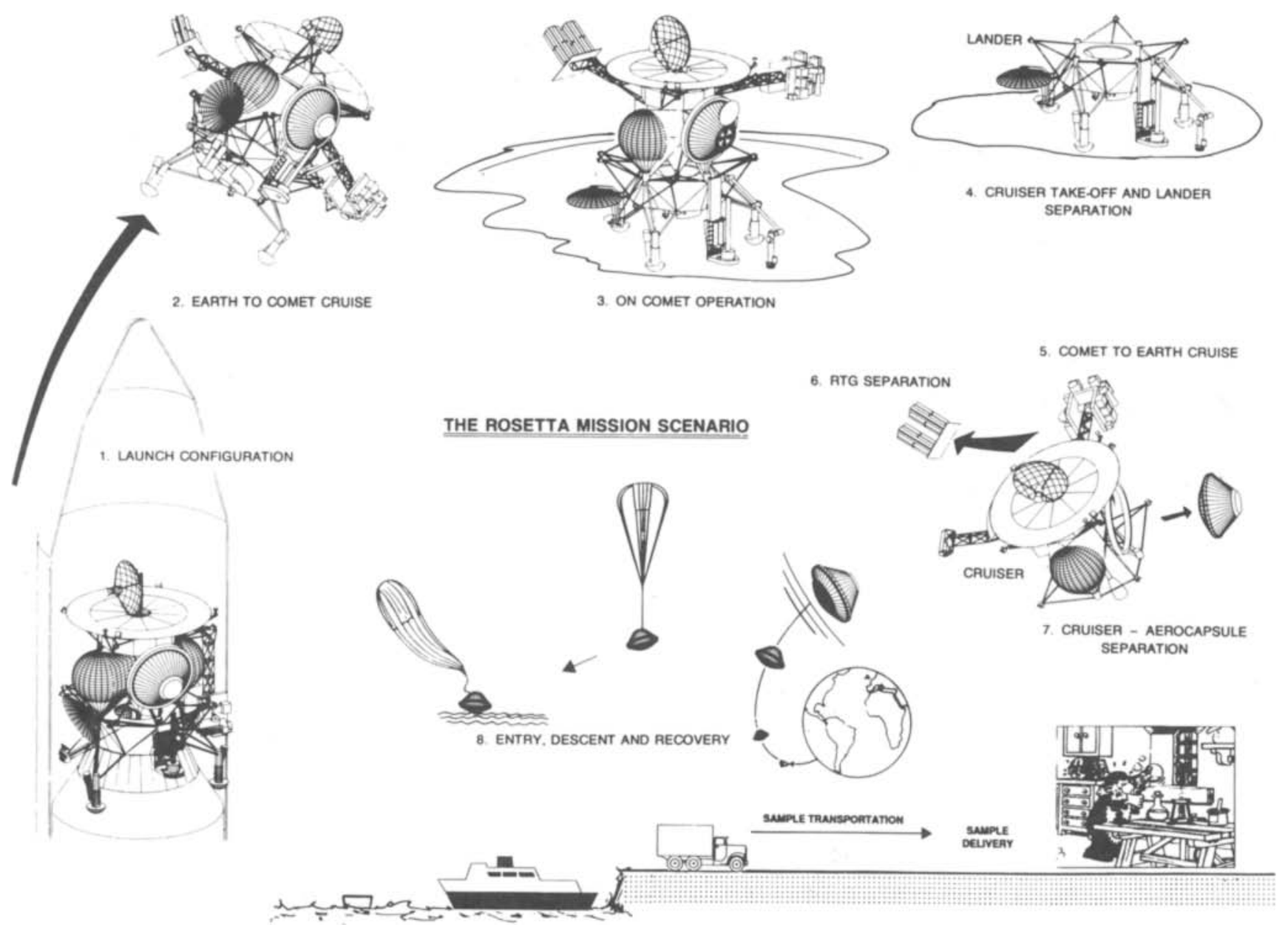

Figure 3. Main phases of the ESA cornerstone mission Rosetta 
plans to make contributions to them. In order to be ready when the large agencies might extend an invitation to participate, studies have been, and are being, performed.

A Mars Exploration Study Team has worked out several scenarios for European participation in Mars explorations. One of them, a global network of remote stations for combined seismic, meteorological, geochemical and geophysical observations, which would be deployed either by semi-hard lander or penetrators, is presently in an assessment study phase. Another study team has started its work to assess possible European contributions to science on and from the moon in the context of a manned lunar base.

In discussing European planetology the national programmes must not be forgotten. They are mainly of the larger countries, can be substantial and are performed on a bilateral basis with the large agencies. Examples are the collaboration of France with the USSR in the Venus and Mars programmes and the German/USA collaborations in the Galileo (Jupiter) and CRAF (Comet Rendez-vous/Asteroid Fly-by) missions.

\section{Conclusion}

I have attempted to demonstrate that the European space science community is capable of making important contributions to solar system science. Although ESA's planetary science programme has just started, what has been achieved and what is under development is quite significant, and the bilateral programmes of several nations are further augmenting Europe's role in this field. ESA, of course, cannot compete in size with NASA and Intercosmos, but its science programme (Horizon 2000) is nevertheless highly interesting, balanced, and competitive, and it can contribute important shares to the large planetary missions. There is no doubt in my mind that Horizon 2000 has given European space scientists a new selfconfidence. For the first time they are acting, rather than reacting to the programmes of the big agencies.

However, this programme could still be jeopardised by financial difficulties, not only within ESA but also in the member countries where the experiments for these missions have to be financed. There is hope that the financial difficulties within the agency will soon be resolved in such a way that the continued implementation of a somewhat stretched Horizon 2000 core programme is secured.

It remains a problem that most ESA member states have not been able to augment their space science budgets in pace with the increase of the ESA science programme. This would be required in order to equip the spacecraft with the necessary advanced instrumentation. There is the additional problem that the Horizon 2000 core programme does not include the more advanced missions to the moon and to Mars.

If, for some reason, ESA and its member states were not able to implement the Horizon 2000 core programme, which is minimal and yet balanced, it would be a disaster. However, I go one step further and claim that Europe could and should also be able to afford an augmented space science programme, including the more advanced missions mentioned above. Not only would its GNP allow it, but it is also well known that the science programmes of the agencies are in the forefront in advancing new technologies. It is in the interests of ESA's other programmes (transportation, telecommunication, and so on) and, more generally, of Europe's economy, for the science programme to be increased relative to these programmes rather than decreased, as it has been in the past.

Considering Europe's cultural heritage in solar system science and after its demonstration with the highly successful Giotto mission, that it has both the technologies and the brains to compete successfully with the large space powers, it would be unforgivable to leave this field to them (and possibly to Japan). I appeal to the members of the Academia Europaea to support within their countries the European Science Programme, which is moderate and yet competitive. 